\title{
Quarkonium Dissociation at Finite Chemical Potential
}

\author{
Uttam Kakade $^{1}$, Binoy Krishna Patra ${ }^{2}$ \\ Department of Physics, Indian Institute of Technology Roorkee, India, 247667
}

\begin{abstract}
We have studied the dissociation of quarkonia states in a deconfined medium of quarks and gluons at the large baryon chemical potential and small temperature region. The aim of this study is to probe the dense baryonic medium expected to be produced at the Facility for Anti-proton and Ion Research (FAIR), GSI Darmstadt. This is done by correcting both the short- and long-distance terms of the Cornell potential by a dielectric function, embodying the effects of deconfined quarks and gluons, at finite baryon chemical potential and temperature. It is found that $J / \psi$ is dissociated approximately at $1.4 \mu_{c}$ in the temperature range $20-50 \mathrm{MeV}$, which can indirectly help to locate the point on the QCD phase diagram at the large chemical potential and low-temperature zone.
\end{abstract}

PACS: $\quad$ 12.39.-x,11.10.St,12.38.Mh,12.39.Pn 12.75.Nq, 12.38.Gc

Keywords: heavy ion collision, QCD phase transition, QGP, nonperturbative effects, quarkonium dissociation

\section{Introduction}

Relativistic heavy-ion collisions provide the unique opportunity of creating a hot and dense nuclear matter in the laboratory. According to statistical quantum chromodynamics (QCD), nuclear matter may undergo a color deconfined partonic phase, the quark gluon plasma (QGP), at sufficiently high temperature and/or density. Over the past few decades, strenuous efforts have been made to devise clean and experimentally viable signals that can unambiguously identify the existence

\footnotetext{
${ }^{1}$ usk11dph@iitr.ac.in, uskakade@gmail.com

${ }^{2}$ binoyfph@iitr.ac.in
} 
of QCD phase transition and trace out its signatures. Charmonium (a bound state of charm and anti-charm quarks) suppression had been predicted as a signature for the deconfinement transition for a long time [1], where the Debye screening of the colored partons was the dominant mechanism of suppression. Experimental investigations revealed a noticeable reduction of the charmonium production in proton-nucleus $(p-A)$ collisions compared to the hadronic collisions (scaled with binary nucleon-nucleon $(p-p)$ collisions) $[2,3,4,5]$. A thorough understanding of this normal nuclear suppression is essential in order to establish a robust baseline reference, with respect to the anomalous suppression $[6,7]$, pertaining to the formation of a deconfined partonic medium observed at Relativistic Heavy Ion Collision (RHIC) and Large Hadron Collider (LHC) energies that mainly scan the high temperature and almost vanishing baryon density region of the QCD phase diagram [8,9]. Depending upon the magnitude of energy deposited in the center-of-mass frame of colliding nuclei, there are in general two scenarios in heavy-ion collision. The first one is possibility of the complete stopping of the colliding nuclei if energy density $\epsilon \approx 5 \mathrm{GeV} / \mathrm{fm}^{3}$, creating sufficiently high baryon density (parton density) to produce QGP. Full transparency of colliding nuclei, the other scenario, takes place if $\epsilon \sim 100 \mathrm{GeV} / \mathrm{fm}^{3}$ creating a favorable environment to produce QGP by vacuum polarization and leading to vanishing chemical potential. Exploration of high baryon densities and the moderate-temperature region of the QCD phase diagram is possible with the upcoming compressed baryonic matter (CBM) experiment at the Facility for Anti-proton and Ion Research (FAIR). At FAIR, the light and heavy ions can be collided in the beam energy range $E_{L a b}=10-40 \mathrm{AGeV}$ to create an environment favorable to investigation of extremely dense nuclear matter in the laboratory through the measurements of bulk and rare probes. Model calculations based on transport and hydrodynamical equations [10] predict that the highest net baryon densities $\left(\rho_{B}\right)$ produced in the center of collision is $\sim 6$ to 12 times the density of normal nuclear matter for the most central collision $(b=0)$.

The FAIR will open avenues to investigate some of the fundamental yet enigmatic issues of strong interaction thermodynamics at large baryon density. Some of the key issues in this region are the study of hadronic properties in dense nuclear matter, the deconfinement phase transition from hadronic to quark-gluon matter driven by high baryon densities, and the nuclear equation of state (EOS) at high baryon densities. Thus the behavior of QCD at high baryon density and moderate to low temperatures is interesting and has potential applications to cosmology, astrophysics of neutron stars, and heavy-ion collisions. Although the motive of FAIR is to produce baryon-rich QGP, it is uncertain that at what point on the phase diagram along the baryon density $\left(\rho_{B}\right)$ axis 
QGP can be produced.

Since the relative velocity of heavy-quark-bound sate $\left(v_{Q \bar{Q}} \ll 1\right)$ is very small, in-medium dynamics of $Q \bar{Q}$ bound states has been extensively studied with phenomenological potential models [11], where the temperature-dependent potential carries all medium effects with nonperturbative terms borrowed from lattice simulations. Although the derivation of such models from QCD is not established, free energies and other quantities [12, 13] from lattice calculations obtained using the correlation functions of the Polyakov loop are often taken as input for the potential. These quantities have been thought to be related to the color-singlet and color-octet heavy quark potentials at finite temperature $[13,14]$ however, a precise answer is still missing [15]. It was until recently that the smallness of the velocity $\left(v_{Q \bar{Q}} \sim \alpha_{s}<1\right)$ has opened up the possibility of studying heavy quark bound sate at finite temperature, where both the non-relativistic scales, viz. the heavy quark mass $\left(m_{Q}\right)$, the momentum exchange $\left(m_{Q} v_{Q}\right)$, the binding energy $\left(m_{Q} v_{Q}^{2}\right)$ etc. and the thermal scales, viz. $T, g T, g^{2} T$ are are hierarchically ordered: By exploiting the aforesaid separation of scales, a sequence of low-energy effective field theories (EFTs) [16, 17] viz. non-relativistic QCD (NRQCD), potential non-relativistic QCD (pNRQCD) etc. have been synthesized by integrating out the successive energy scales. In this context, pNRQCD and its thermal version are of particular interest because it describes the quarkonium dynamics through potentials and low-energy interactions $[18,19]$. The color-screening phenomenon [18] and thermal width are thought to be an outcome of thermal corrections to the real and imaginary parts of the color-singlet potential, respectively. Out of various mechanisms that might be contributing to the imaginary part of the potential, Landau-damping [20] is the dominant one, which is responsible for quarkonium dissociation at weak coupling. It makes the quarkonium to be dissociated even at temperatures, where the possibility of color screening is negligible. Moreover, lattice studies have also shown that even at strong coupling $[21,22,23]$ the potential may have a sizeable imaginary part and such contributions are related to quarkonium decay processes in the plasma.

The works referred above are restricted to finite temperature only. However, the color screening effect at finite temperature and finite density has been studied in thermo-field dynamics approach to calculate the Debye screening mass, $m_{D}(T, \mu)$, where authors in Refs. [24] and [25] have used the phenomenological potential model [11] and error function-type confined force with color screened Coulomb-type potential, respectively. Applying the effective perturbation theory for gauge theo- 
ries at finite temperature [26], authors in Ref.[27] extended it to the finite chemical potential for studying the collisional energy-loss of heavy quark in QGP. Photon production at finite chemical potential in QCD plasma at leading-order in strong coupling has been computed in [28]. Furthermore the dissipative hydrodynamic effects on QGP at finite density has been studied by developing a causal dissipative hydrodynamic model at finite baryon density for RHIC and LHC energies to study the net-baryon rapidity distribution [29]. Although, finite density lattice QCD calculations are seriously affected by the sign problem, recently color screening in heavy quark potential at finite density with Wilson fermions has been studied in lattice QCD in both real and imaginary chemical potential regions [30].

In the present work we have studied quarkonium dissociation in the quark matter of high baryon density and low temperature. For that we have first obtained the heavy quark potential by correcting both perturbative and nonperturbative terms in the Cornell potential through the dielectric function at finite temperature and chemical potential and then calculate the binding energy of the quarkonium states. The rest of the work is organized as follows: We first discuss the heavy quark potential at finite chemical potential in Sec.II. Then we focus on the estimation of the binding energy of quarkonium at finite chemical potential and thereby obtain the dissociation chemical potential for quarkonium ground states in Sec.III. Finally we conclude in Sec.IV.

\section{Heavy quark potential at finite chemical potential and running coupling constant}

The interaction potential between $Q$ and $\bar{Q}$ is subjected to modification when the pair $(Q \bar{Q})$ is placed in a hot and dense QCD medium, and it plays an important role in understanding the status of $Q \bar{Q}$ bound states in such medium. This issue has been well taken up by various authors and is reported in several reviews [31, 32], by dealing on both fronts, the phenomenology [11] as well as the lattice QCD [21, 22, 23, 33]. All these studies assume that the phase transition from a hadronic matter to a QGP phase melts the string, and thereby the string tension vanishes at the transition point. However, lattice results [34] indicate that there is no genuine phase transition at vanishing baryon density, it is rather a crossover, and there may not be immediate melting of string at the deconfinement temperature. Thus one should consider its effect on the potential even above the transition temperature, in addition to the coulomb term. 
The large mass of heavy quark meets the requirements: (i) $m_{Q} \gg \Lambda_{Q C D}$ and (ii) $T \ll m_{Q}$ for the description of the interactions between $Q \bar{Q}$ at finite temperature and density. It is thus possible to obtain the quantum mechanical potential $\mathrm{V}(\mathrm{r}, \mathrm{T})$, by correcting short as well as longdistance parts of the $Q \bar{Q}$ potential through dielectric permittivity, $\epsilon(k)$, embodying the effects of deconfined medium:

$$
V(r, T, \mu)=\int \frac{d^{3} \mathbf{k}}{(2 \pi)^{3 / 2}}\left(e^{i \mathbf{k} \cdot \mathbf{r}}-1\right) \frac{V(k)}{\epsilon(k)} .
$$

The term $V(k)$ in above equation is the Fourier Transform (FT) of the potential. However, obtaining the FT of the linear term is a tricky part and needs regulation, which is carried out by multiplying an exponential damping factor and is then switched off after the evaluation of FT. We have regulated both terms by the same screening scale ${ }^{3}$ and the FT of the Cornell potential is thus obtained as [35]

$$
\mathbf{V}(k)=-\frac{\sqrt{2 / \pi}}{k^{2}}\left(\alpha+\frac{2 \sigma}{k^{2}}\right),
$$

where $\alpha$ and $\sigma$ are the coupling and string tension, respectively. We have taken $\alpha$ as function of chemical potential $\left(\mu_{q}\right)$ and temperature up to two loops [36] as

$$
\alpha\left(T, \mu_{q}\right)=\frac{6 \pi}{\left(33-2 N_{f}\right) \ln \sqrt{\frac{T^{2}}{\Lambda_{T}^{2}}+\frac{\mu_{q}^{2}}{\pi^{2} \Lambda_{T}^{2}}}}\left[1-\frac{3\left(153-19 N_{f}\right) \ln \left(2 \ln \sqrt{\frac{T^{2}}{\Lambda_{T}^{2}}+\frac{\mu_{q}^{2}}{\pi^{2} \Lambda_{T}^{2}}}\right)}{\left(33-2 N_{f}\right)^{2} \ln \sqrt{\frac{T^{2}}{\Lambda_{T}^{2}}+\frac{\mu_{q}^{2}}{\pi^{2} \Lambda_{T}^{2}}}}\right],
$$

where $\Lambda_{T}$ is the QCD scale-fixing parameter which characterizes the strength of the interaction. To obtain the dielectric permittivity, $\epsilon(k)$, one has to evaluate the self-energies and the corresponding static propagators in weak coupling Hard Thermal Loop (HTL) approximation. In the realtime formalism using Keldysh representation, the retarded (R), advanced (A) and symmetric (F) propagators are written as the linear combination of the components of the $(2 \times 2)$ matrix propagator:

$$
D_{R}^{0}=D_{11}^{0}-D_{12}^{0}, D_{A}^{0}=D_{11}^{0}-D_{21}^{0}, D_{F}^{0}=D_{11}^{0}+D_{22}^{0},
$$

where only the symmetric component involves the distribution functions and is of particular advantage for the HTL diagrams, where the terms containing distribution functions dominate. Similar relations hold good for the retarded $\left(\Pi_{R}\right)$, advanced $\left(\Pi_{A}\right)$ and symmetric $\left(\Pi_{F}\right)$ self energies. The

\footnotetext{
${ }^{3}$ However, in the framework of classical Debye-Hückel theory, different screening functions were employed, viz. $f_{c}$ and $f_{s}$ for the Coulomb and string terms, respectively to obtain the free energy [37] whereas in Ref. [38], different scales for the Coulomb and linear pieces have been employed through a dimension-two gluon condensate.
} 
resummation of the propagators can be obtained using the Dyson-Schwinger equation

$$
\begin{aligned}
D_{R, A} & =D_{R, A}^{0}+D_{R, A}^{0} \Pi_{R, A} D_{R, A} \\
D_{F} & =D_{F}^{0}+D_{R}^{0} \Pi_{R} D_{F}+D_{F}^{0} \Pi_{A} D_{A}+D_{R}^{0} \Pi_{F} D_{A} .
\end{aligned}
$$

For the static potential, we need only the temporal component ("00" $\equiv$ L) of the propagator, whose evaluation is easier in the Coulomb gauge. Thus the above resummation (5) can be recast through its temporal component as

$$
D_{R, A}^{L}=D_{R, A}^{L(0)}+D_{R, A}^{L(0)} \Pi_{R, A}^{L} D_{R, A}^{L}
$$

The leading contribution to temporal component of the retarded, advanced and symmetric gluon self-energy in the HTL-approximation can be written as

$$
\Pi_{R, A}^{L}(k)=m_{D}^{2}\left(\frac{k_{0}}{2 k} \ln \frac{k_{0}+k \pm i \epsilon}{k_{0}-k \pm i \epsilon}-1\right)
$$

and

$$
\Pi_{F}^{L}(k)=-2 \pi i m_{D}^{2} \frac{T}{k} \Theta\left(k^{2}-k_{0}^{2}\right)
$$

respectively. Thus the gluon self-energy is composed of real and imaginary parts which are responsible for the Debye screening and the Landau damping, respectively. The real part of potential can be obtained from the retarded (or advanced) self energy with the prescriptions $+i \epsilon(-i \epsilon)$, respectively.

The term $m_{D}$ in above equation is known as electric screening mass (Debye screening mass) which encodes the medium effects in terms of temperature and baryon chemical potential. The properties of QCD at finite temperature and density are studied in [39] and the electric screening mass can be obtained in the static infra-red limit of " 00 " component of gluon self energy $\Pi^{00}\left(q_{0}=0, \vec{q} \rightarrow 0\right)$. Taking the dynamical quark mass $m_{q}=0$, the electric screening mass at finite temperature and vanishing baryon chemical potential $\left(\mu=\mu_{b}=0\right)$ based on perturbation theory in high temperature limit is [40],

$$
\Pi^{00}\left(q_{0}=0, \vec{q} \rightarrow 0\right)=\left(\frac{N_{c}}{3}+\frac{N_{f}}{6}\right) g T,
$$

where $N_{c}$ and $N_{f}$ are number of colors and flavours of color group $\mathrm{SU}\left(N_{c}\right)$ respectively. For a hot and dense plasma, the resummation technique to the perturbation theory proposed by [26] works well in momentum scale $g T$. The leading-order Debye screening mass from HTL $[27,41,42,43]$ 
at finite temperature and chemical potential is given by

$$
m_{D}^{2}\left(T, \mu_{q}\right)=g^{2}(T) T^{2}\left(\frac{N_{c}}{3}+\frac{N_{f}}{6}+\frac{1}{2 \pi^{2}} \sum_{f} \frac{\mu_{q}^{2}}{T^{2}}\right)
$$

where the quark chemical potential is related to the baryon chemical potential by $\mu_{q}=\mu_{b} / 3$. Later the screening mass at finite temperature and baryon density has also been studied on the lattice through the Taylor expansion method [44], which coincides with the above equation. For zero chemical potential $\left(\mu_{q}=0\right)$, the leading-order Debye mass $(11)$ reduces to

$$
m_{D}(T)=g(T) T \sqrt{\frac{N_{c}}{3}+\frac{N_{f}}{6}} .
$$

The next-to-leading contribution to Debye mass $\left(\delta m_{D}\left(T, \mu_{q}\right)\right)$ comes from the resumed oneloop gluon diagrams [26]. However, we restrict ourselves only up to the leading-order term in the Debye mass. The variations of the Debye mass in leading-order with the chemical potential and temperature are shown in Fig. 1. The left panel of Fig.1 shows that the Debye mass increases with the chemical potential and is more for higher temperature. This implies that the contribution to the Debye mass at low temperature and high baryon chemical potential mainly comes from the chemical potential, which can be seen in the right panel of Fig.1. For low temperature region (20$50 \mathrm{MeV}$ ) the increase in Debye mass is negligible but increases with $\mu_{b}$. The temporal component of the retarded (or advanced) propagator in the static limit [45] is

$$
D_{R, A}^{00}(0, k)=-\frac{1}{\left(k^{2}+m_{D}^{2}(T, \mu)\right)},
$$

Hence we can now obtain the dielectric permittivity from the static limit of the "00"-component of gluon propagator

$$
\epsilon^{-1}(k)=-\lim _{\omega \rightarrow 0} k^{2} D_{11}^{00}(\omega, k) ; \quad D_{11}^{00}(\omega, k)=\frac{1}{2}\left(D_{R}^{00}+D_{A}^{00}\right)
$$

Thus the medium modified potential is obtained by substituting Eqs. (2) and (14) into Eq.(1)

$$
V(\mathbf{r}, T, \mu)=\int \frac{d^{3} \mathbf{k}}{(2 \pi)^{3 / 2}}\left(e^{i \mathbf{k} \cdot \mathbf{r}}-1\right)\left(-\frac{\sqrt{2 / \pi}}{k^{2}}\left(\alpha+\frac{2 \sigma}{k^{2}}\right)\right)\left[\frac{k^{2}}{\left(k^{2}+m_{D}^{2}(T, \mu)\right)}\right]
$$

Solving the integral in above equation, one can to obtain the medium modified potential at finite 

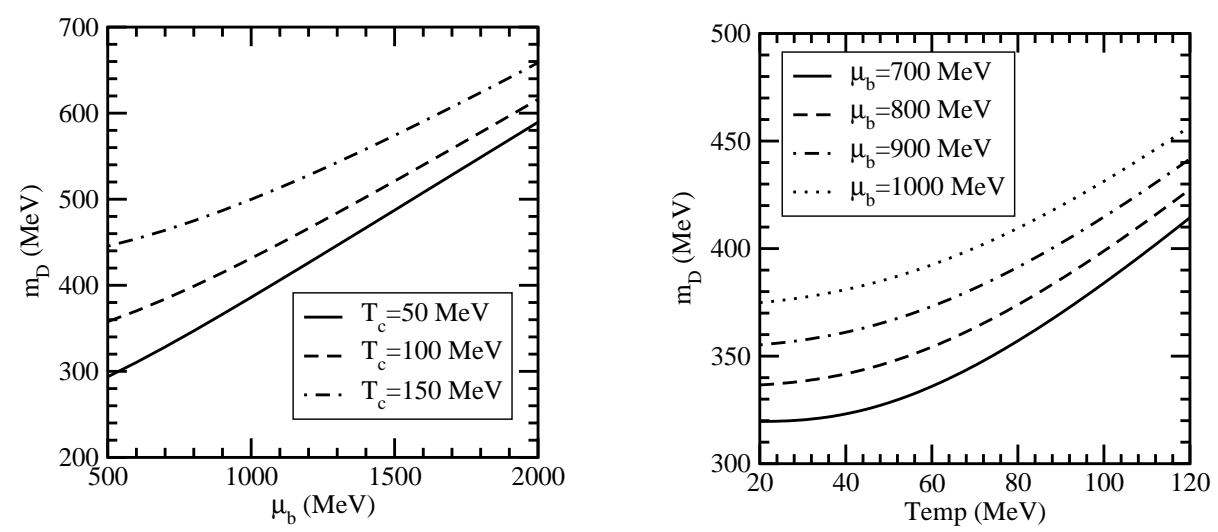

Figure 1: Variation of the screening mass $\left(m_{D}\right)$ against the baryon chemical potential $\left(\mu_{b}\right)$ at different values of temperature (left panel). Same against the temperature at different values of baryon chemical potential (right panel).

density and temperature (with $\hat{r}=r m_{D}(T, \mu)$ ) as:

$$
\mathbf{V}(r, T, \mu)=\frac{2 \sigma}{m_{D}(T, \mu)}\left(\frac{e^{-\hat{r}}-1}{\hat{r}}+1\right)-\alpha m_{D}(T, \mu)\left(\frac{e^{-\hat{r}}}{\hat{r}}+1\right)
$$

where the chemical potential and temperature dependencies are introduced through the Debye mass, $m_{D}(T, \mu)$. The potential has a long range Coulombic tail in addition to the standard Yukawa term and the constant terms are introduced to yield the correct limit of $V(r, T, \mu)$ as $T \rightarrow 0, \mu \rightarrow 0$ (it reduces to the Cornell potential).

Recently it is known that there exists imaginary part of the potential $[16,17,21,46]$ which originates from the static limit of symmetric self energy (9) and plays an important role in weakening the bound state peak that leads to a finite width $(\Gamma)$ for the resonance peak in the spectral function. The imaginary part of the temporal component of symmetric propagator gives the corresponding dielectric function [45]

$$
\epsilon^{-1}(0, k)=\frac{-\pi T m_{D}^{2}(T, \mu) k^{2}}{k\left(k^{2}+m_{D}^{2}(T, \mu)\right)^{2}}
$$

which, in turn, gives the imaginary part of $Q \bar{Q}$ potential:

$$
\begin{aligned}
\Im V(\mathbf{r}, T, \mu) & =-\int \frac{d^{3} \mathbf{k}}{(2 \pi)^{3 / 2}}\left(e^{i \mathbf{k} \cdot \mathbf{r}}-1\right)\left(-\frac{\sqrt{2 / \pi}}{k^{2}}\left(\alpha+\frac{2 \sigma}{k^{2}}\right)\right) k^{2}\left[\frac{-\pi T m_{D}^{2}(T, \mu)}{k\left(k^{2}+m_{D}^{2}(T, \mu)\right)^{2}}\right] \\
& =-\alpha T \phi_{0}(\hat{r})+\frac{2 \sigma T}{m_{D}^{2}(T, \mu)} \psi_{0}(\hat{r}),
\end{aligned}
$$


where the first (second) term comes from contribution due to the Coulomb (linear) term in the leading-order $[47,48,49]$ with the following functions, $\phi_{0}(\hat{r})$ and $\psi_{0}(\hat{r})$, respectively:

$$
\begin{aligned}
& \phi_{0}(\hat{r})=-\frac{\hat{r}^{2}}{9}\left(-4+3 \gamma_{E}+3 \log \hat{r}\right) \\
& \psi_{0}(\hat{r})=\frac{\hat{r}^{2}}{6}+\frac{-107+60 \gamma_{E}+60 \log (\hat{r})}{3600} \hat{r}^{4}+O\left(\hat{r}^{5}\right) .
\end{aligned}
$$

Thus in leading-logarithmic order, the imaginary component of potential becomes

$$
\Im V(r, T, \mu)=-T\left(\frac{\alpha \hat{r}^{2}}{3}+\frac{\sigma \hat{r}^{4}}{30 m_{D}^{2}(T, \mu)}\right) \log \left(\frac{1}{\hat{r}}\right),
$$

which shows that imaginary part vanishes for small distances [45]. However, its magnitude is larger than the case where only the Coulombic term is considered $[47,50,51]$, due to the additional linear (string) term.

Now with the imaginary potential (21), the width for a particular resonance state can be calculated as:

$$
\begin{aligned}
\Gamma(T, \mu) / 2 & =\int d^{3} \mathbf{r}|\Psi(r)|^{2}\left[\frac{\alpha T \hat{r}^{2}}{3}+\frac{T \sigma \hat{r}^{4}}{30 m_{D}^{2}(T, \mu)}\right] \log (1 / \hat{r}) \\
& =T\left(\frac{4}{\alpha m_{Q}^{2}}+\frac{12 \sigma}{\alpha^{2} m_{Q}^{4}}\right) m_{D}^{2} \log \frac{\alpha m_{Q}}{2 m_{D}(T, \mu)} .
\end{aligned}
$$

Here for simplicity, we have taken $\Psi(r)$ as the Coulombic wave function, similar to the ground state wave function in hydrogen atom. This shows that $\Gamma$ is function of both chemical potential and temperature through the screening mass (Debye mass). However, the dependences on temperature and chemical potential are opposite, namely $\Gamma$ increases with temperature but decreases with chemical potential. That is why $\Gamma$ does not have much role in dissociation of quarkonia states unlike at high temperature. This will be discussed in the next section.

It is worth to note that the potential in a hot QCD medium is not the same as the lattice parametrized heavy quark free-energy in the deconfined phase which is basically a screened Coulomb [52] because one-dimensional Fourier transform of the Cornell potential in the medium yields the similar form as used in the lattice QCD to study the quarkonium properties which assumes the one-dimensional color flux tube structure [53]. However, at finite temperature and density that may not be the case since the flux tube structure may expand in more dimensions [52], hence the three-dimensional form of the medium modified Cornell potential may therefore be the better option. The potential (16) leads to an analytically solvable Coulomb potential if one neglect Yukawa term in the limit $r>>1 / m_{D}$ and the product $\alpha m_{D}$ will be much greater than $2 \sigma / m_{D}$ 

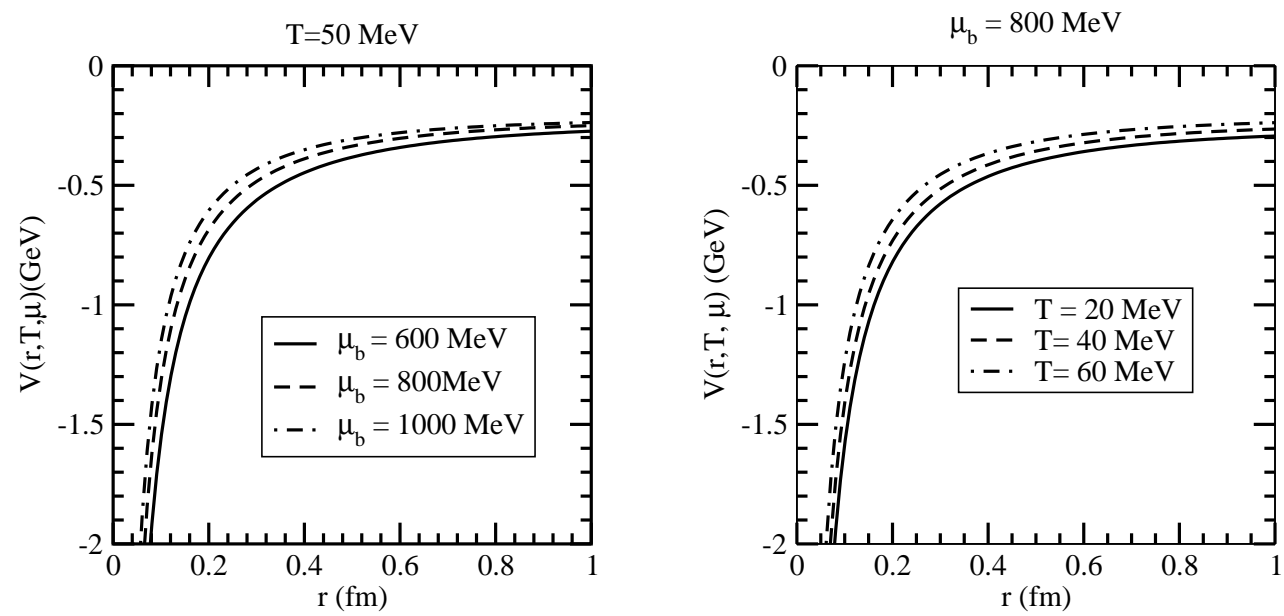

Figure 2: Variation of medium modified $Q \bar{Q}$ potential in a hot and dense QCD medium at given low temperature with different baryon chemical potential $\mu_{b}$ (left panel). Same for different temperatures at given $\mu_{b}$ (right panel).

for large values of chemical potentials.

$$
V(r, T, \mu) \sim-\frac{2 \sigma}{m_{D}^{2}(T, \mu) r}-\alpha m_{D}(T, \mu)
$$

To see the effects of a hot and dense medium on the $Q \bar{Q}$ potential, we have evaluated the potential at large chemical potential, viz. at $600 \mathrm{MeV}, 800 \mathrm{MeV}$ and $1000 \mathrm{MeV}$ in the low temperature range 20-60 MeV in Fig.2. We have found that the potential rises with the interquark distance, $r$, but slower than linearly, i.e. long-range QCD force becomes short-range and at some large enough $r$, it simply flattens out due to the breaking of QCD string, i.e. a heavy meson splits into two heavy-light mesons. This observation agrees with the lattice calculations $[54,55]$. Thus, the deconfinement is reflected in the large-distance behaviour of heavy quark potential even at large chemical potential. That is why the in-medium behaviour of heavy quark bound states is used to probe the state of matter in QCD thermodynamics at finite density and/or temperature.

\section{Binding energy and dissociation chemical potential}

The medium-modified effective potential obtained in the previous section has been employed to study the in-medium properties of the heavy quark bound states such as, binding energies and dissociation chemical potentials. We studied the binding energy and the dissociation chemical potential for the ground states of $c \bar{c}$. In order to understand bound state properties of quarkonium 
states in hot and dense QCD medium, one need to solve the Schrödinger equation using the $Q \bar{Q}$ potential. It can be seen from Eq.(16) that in the short-distance limit, the vacuum contribution of $Q \bar{Q}$ potential supersedes over the in-medium contribution whereas the in-medium contribution affects the potential in the long-distance limit. As we have already seen the potential (23) in long-distance $\left(r m_{D} \gg 1\right)$ limit and for large values of chemical potential (or temperature) is Coulomb-like potential after identifying $2 \sigma / m_{D}^{2}$ with the square of color charge, $g_{s}^{2}$, so the energy eigenvalues are read as in hydrogen atom problem:

$$
E_{\text {bin }} \stackrel{\hat{r} \gg 1}{=}\left(\frac{\sigma m_{Q}}{m_{D}^{2}(T, \mu) n^{2}}+\alpha m_{D}(T, \mu)\right) ; n=1,2 \cdots
$$

However, in the intermediate-distance $\left(r m_{D}(T, \mu) \simeq 1\right)$ scale, the interaction becomes nontrivial and the potential does not look simpler in contrast to the asymptotic limits, thus the full potential (16) in general needs to be dealt numerically to obtain the binding energies. The matrix method serves the purpose and the stationary Schrödinger equation can be solved in a matrix form through a discrete basis, instead of the continuous real-space position basis spanned by the states $|\vec{x}\rangle$. Subdividing the potential $V(16)$ into N discrete wells having potentials $V_{1}$ through $V_{N+2}$, such that $V=V_{i}$ for $x_{i-1}<x<x_{i} ; i=2,3, \ldots,(N+1)$ is the $i^{\text {th }}$ boundary potential. For existence for a bound state, there must be an exponentially decaying wave function in the region $x>x_{N+1}$ as $x \rightarrow \infty$ and the wave function in this region reads:

$$
\Psi_{N+2}(x)=P_{E} \exp \left[-\gamma_{N+2}\left(x-x_{N+1}\right)\right]+Q_{E} \exp \left[\gamma_{N+2}\left(x-x_{N+1}\right)\right]
$$

where, $P_{E}=\frac{1}{2}\left(A_{N+2}-B_{N+2}\right), Q_{E}=\frac{1}{2}\left(A_{N+2}+B_{N+2}\right)$ and, $\gamma_{N+2}=\sqrt{2 \mu_{r}\left(V_{N+2}-E\right)}$. The energy eigenvalues can then be identified with the zero's of $Q_{E}$.

Thus, matrix method gives the binding energies as a function of chemical potential in a small temperature range $(20-40 \mathrm{MeV})$, which are shown in Fig. 3. It is found that the binding energy decreases with the increase of chemical potential. This can be understood by the fact that the screening gets stronger with the increase of the baryon chemical potential and hence the interquark potential becomes weaker compared to $\mu=0$. Thus the study of the binding energies at finite temperature and chemical potential provides a information about the dissociation pattern of quarkonium states in the region of high baryon chemical potential and low temperature.

Now one can obtain the dissociation chemical potential of quarkonia when their binding energies in the dense baryonic medium are of the order of the baryon chemical potential. We found that with the rise in temperature, the dissociation chemical potential deceases (Table 1) slightly, for 


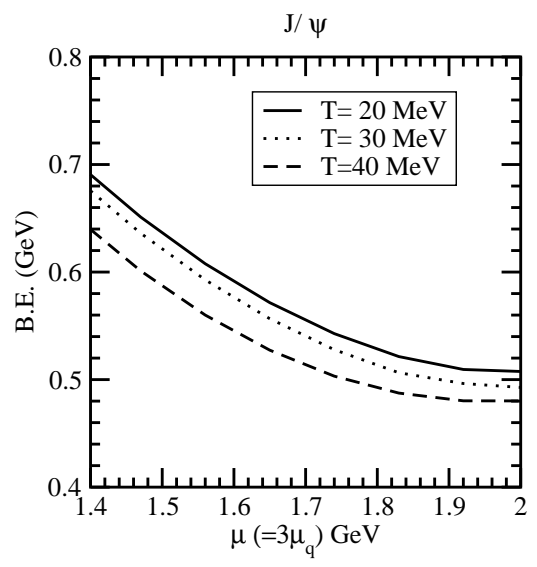

Figure 3: Variation of binding energy of $J / \psi$ state in a hot/dense QCD medium with baryon chemical potential at different values of temperature.

\begin{tabular}{|c|c|c|}
\hline$T(\mathrm{MeV})$ & $\mu_{D}(\mathrm{MeV})$ & $\mu_{D} / \mu_{c}$ \\
\hline 20 & 1683 & 1.42 \\
\hline 30 & 1659 & 1.40 \\
\hline 40 & 1611 & 1.38 \\
\hline 50 & 1560 & 1.35 \\
\hline
\end{tabular}

Table 1: Dissociation chemical potential for $J / \psi$ at different temperatures.

example, at temperature $T=20 \mathrm{MeV}$, the $J / \psi$ will be dissolved at $1.42 \mu_{c}$ whereas for $\mathrm{T}=30 \mathrm{Mev}$, $\mu_{D}$ becomes $1.40 \mu_{c}^{4}$ etc. This observation can be used to locate the point on QCD phase diagram in the high-baryon density limit where the confined nuclear matter cross to the phase boundary and becomes baryon-rich deconfined quark matter Recently the physics of dissociation has been further complicated by arguing that the potential should have an imaginary part as well, which, like a width, facilitates the dissociation of quarkonia further. Thus, nowadays the dissociation temperatures are obtained from the width, $\Gamma$, where the dissociation is expected to occur when binding energy decreases with the chemical potential and becomes $\sim \Gamma / 2[56,57]$. We have found that the dissociation chemical potential estimated from the above criterion is almost same to the values obtained from the binding energy criterion (Table 1).

\footnotetext{
${ }^{4}$ The values of $T_{c}$ and $\mu_{c}$ used in this work are taken from the Ref. [58], viz. the critical baryon chemical potentials $\left(\mu_{c}\right)$ are $1189 \mathrm{MeV}, 1183 \mathrm{MeV}, 1169 \mathrm{MeV}$ and $1154 \mathrm{MeV}$ for the critical temperatures $\left(T_{c}\right) 20 \mathrm{MeV}, 30$ $\mathrm{MeV}, 40 \mathrm{MeV}$ and $50 \mathrm{MeV}$, respectively.
} 


\section{Conclusion}

We have studied the quarkonium dissociation in hot and dense QCD medium by correcting both the perturbative and nonperturbative terms of the Cornell potential through the dielectric permittivity Thereafter the chemical potential- and temperature-dependent potential is plugged into Schrödinger equation to study the properties of quarkonia states in high baryon density and low temperature region.

It is noticed that the screening mass increases dominantly with the chemical potential rather than with the temperature in the high baryon density and low temperature region. As a consequence the binding energies decrease with the chemical potential. The dissociation chemical potentials of $J / \psi$ at $T=40$ and $50 \mathrm{MeV}$ is found to be $1611 \mathrm{MeV}$ and $1560 \mathrm{MeV}$, respectively. Finally, we conclude that the dissociation of quarkonia states in large baryon density may help to trace out the point on the QCD phase diagram at which the baryon rich QGP (which is expected at FAIR energies) will be created.

Acknowledgement: We are thankful to Lata Thakur for her sincere help in deriving the quarkonium potential. B.K.P. is thankful to Government of India for financial assistance under CSIR project (CSR-656-PHY). Uttam Kakade is thankful to the Government of Maharashtra for availing study leave.

\section{References}

[1] T. Matsui and H. Satz, Phys. Lett. B 178, 416 (1986).

[2] B. Alessandro et.al (NA 50 collab), Eur.Phys. J. C 39, 335 (2005).

[3] J. P. Blaizot, Phuong Mai Dinh, Jean-Yves Ollitrault, Phys. Rev. Lett. 85, 4012 (2000).

[4] L. Grandchamp, R. Rapp, G. E. Brown, Phys. Rev. Lett. 92, 212301 (2004).

[5] R. Arnaldi et. al. NA 50 Collaboration, Phys. Rev. Lett. 99, 132302 (2007).

[6] M. C. Abreu et al., (NA50 Coll.), Phys. Lett. B 410, 337 (1997). 
[7] B. Alessandro et al. (NA50 Coll.), Eur. Phys. J. C 48, 329 (2006).

[8] Dipali Pal, Binoy Krishna Patra, D. K. Srivastava, Eur.Phys.J.C17:179 (2000).

[9] Binoy Krishna Patra, Dinesh Kumar Srivastava, Phys. Lett. B505,113 (2001).

[10] I. C. Arsene et al., Phys. Rev. C 75, 034902 (2007).

[11] F. Karsch, M. T. Mehr and H. Satz, Z. Phys. C 37, 617 (1988).

[12] L. D. McLerran and B. Svetitsky, Phys. Rev. D 24, 450 (1981).

[13] S. Nadkarni, Phys. Rev. D. 34, 3904 (1986).

[14] S. Nadkarni, Phys. Rev. D 33, 3738 (1986).

[15] O. Philipsen, Nucl. Phys. A 820, 33C (2009).

[16] M. A. Escobedo and J. Soto, Phys. Rev. A 78, 032520 (2008).

[17] N. Brambilla, J. Ghiglieri, A. Vairo and P. Petreczky, Phys. Rev. D 78, 014017 (2008).

[18] A. Pineda and J. Soto, Nucl. Phys. Proc. Suppl. 64, 428 (1998).

[19] N. Brambilla, A. Pineda, J. Soto and A. Vairo, Nucl. Phys. B 566, 275 (2000).

[20] M. Laine, O. Philipsen, P. Romatschke, and M. Tassler, JHEP 0703, 054 (2007).

[21] M. Laine, O. Philipsen, and M. Tassler, JHEP 09, 066 (2007).

[22] A. Rothkopf, T. Hatsuda and S. Sasaki, Phys. Rev. Lett. 108, 162001 (2012).

[23] Y. Burnier and A. Rothkopf, Phys. Rev. D 86, 051503 (2012).

[24] S. Gao, B. Liu and W. Q. Chao, Phys. Lett. B 378, 23 (1996).

[25] B. Liu ,P. N. Shen and H. C. Chiang, Phys. Rev. C 55,3021 (1997).

[26] E. Braaten, R. D. Pisarski, Nucl. Phys. B 337, 569 (1990).

[27] Hans Vija and M. H. Thoma, Phys. Lett. B 342, 121 (1995).

[28] Hualong Gervais and Sangyong Jeon, Phy. Rev. C 86, 034904 (2012). 
[29] Akhihiko Monnai, Journal of Physics:Conference Series 432, 012011 (2013).

[30] Junichi Takahashi et al., Phys. Rev. D 88, 114504 (2013).

[31] N. Brambilla, et al., Rev. Mod. Phys. 77,1423 (2005).

[32] L. Kluberg and H. Satz, arXiv:hep-ph/0901.3831.

[33] E. Laermann et. al Phys. Lett. B 173, 437 (1988).

[34] F. Karsch, J. Phys.: Conf. Series 46, 122 (2006).

[35] V. Agotiya, V. Chandra and B. K. Patra, Phys. Rev. C 80, 025210 (2009).

[36] P. K. Srivastava, S. K. Tiwari and C. P. Singh, Phys. Rev. D 82, 014023 (2010).

[37] S. Digal, O. Kaczmarek, F. Karsch and H. Satz, Eur. Phys. J. C 43, 71 (2005).

[38] E. Megias, E. Ruiz Arriola, L.L. Salcedo, Phys. Rev. D 75, 105019 (2007).

[39] K. Kajantie and J. K. Kapusta, Ann. Phys.(N. Y.) 160, 477 (1985).

[40] O. K. Kalashnikov, Fortschr.Phy 32, 525 (1984).

[41] E. Braaten, R. D. Pisarski, Phy. Rev. D 42, 2156 (1990);Phys. Rev. Lett. 64, 1338 (1990).

[42] R. D. Pisarski, Nucl. Phys. A 525, 175 (1991).

[43] R. D. Pisarski, Phy. Rev. D 47, 5589 (1993).

[44] M. Doring, S. Ejiri, O. Kaczmarek, F. Karsch and E. Laermann, PoS LAT 2005-005, 193 (2006) [heplat/0509150].

[45] Lata Thakur, Uttam Kakade and Binoy Krishna Patra, Phys. Rev. D 89, 094020 (2014).

[46] A. Beraudo, J. P. Blaizot, C. Ratti, Nucl. Phys. A bf 806, 312 (2008).

[47] A. Dumitru, Y. Guo and M. Strickland, Phys. Rev. D 79, 114003 (2009).

[48] A. Dumitru, Y. Guo, A. Mocsy and M. Strickland, Phys. Rev. D 79, 54019 (2009).

[49] M. Margotta, K. McCarty, C. McGahan, M. Strickland and D. Yager-Elorriaga, Phys. Rev. D 83, 105019 (2011). 
[50] A. Dumitru, Y. Guo and M. Strickland, Phys. Lett. B 662, 37 (2008).

[51] Y. Burnier, M. Laine and M. Vepsalainen, Phys. Lett. B 678, 86 (2009)[arXiv:0903.3467].

[52] H. Satz, Rept. Prog. Phys. 63, 1511 (2000).

[53] Vijai V. Dixit, Mod. Phys. Lett. A 5, 227 (1990).

[54] O. Kaczmarek S. Ejiri, F. Karsch, E. Laermann and F. Zantow, Prog. Theor. Phys. Supl.153, 287 (2004).

[55] Y. Burnier, O. Kaczmarek and A. Rothkopf, Phys. Rev. Lett. 114, 082001 (2015).

[56] A. Mocsy and P. Petreczky, Phys. Rev. Lett.99, 211602 (2007).

[57] Y. Burnier, M. Laine, and M. Vepsalainen, J. High Energy Phys.01, 043 (2008).

[58] J. I. Kapusta, Phys. Rev. C 81, 055201 (2010)[arxiv:1001.3650v1, nucl-th]. 